\title{
Unusual primary location of the malignant melanoma, the cervico-vaginal region: 3 cases and literature review ${ }^{*}$
}

\author{
El Fatemi Hinde' ${ }^{1}$ Bennani Amal ${ }^{1}$, Saadi Hannane², Bouchikhi Cherhazade ${ }^{2}$, Bannani Abdelaziz ${ }^{2}$, \\ Mellas Nawfel $^{3}$, Mesbahi Omar ${ }^{3}$
}

${ }^{1}$ Department of Pathology, Hassan II Teaching Hospital, Fez, Morocco

${ }^{2}$ Department of Gynecology, Hassan II Teaching Hospital, Fez, Morocco

${ }^{3}$ Department of Oncology, Hassan II Teaching Hospital, Fez, Morocco

Email: Hinde0012@hotmail.com

Received 4 July 2012; revised 8 August 2012; accepted 20 August 2012

\begin{abstract}
Introduction: Malignant melanoma (MM) represents $1 \%$ of all cancers and has an incidence of $3 \%-7 \%$ in the female genital tract, the majority of cases being reported in the vulva. The cervico-vaginal region is an unusual primary location of the malignant melanoma. Case report: The clinical and morphologic findings of 3 MM patients with cervico-vaginal tumours mimicking cervical polyp in third case. Histologically, the neoplasia was formed by nodules of broad cytoplasmic cells with poorly defined borders with pleomorphic nuclei with prominent nucleolar. Tumor cells were positive for S-100 protein, Melan A, HMB 45 and CD117. The authors discuss three cases of cervico-vaginal melanoma and analyze clinical and prognostic aspects of this disease. Conclusion: Primary MM of the cervix should be considered in the differential diagnosis of cervical malignancies. Early diagnosis is essential in order to warrant a better prognosis, although there are no cases of cure described.
\end{abstract}

Keywords: Genital Tract; Malignant Melanoma; Cervix; Vagina; Chemotherapy; CD117

\section{INTRODUCTION}

Mucosal melanoma is a rare cancer that is clearly distinct from its cutaneous counterpart in biology, clinical course, and prognosis. Recent studies have shown important differences in the frequencies of various genetic alterations in different subtypes of melanoma. Activating mutations in the c-KIT gene are detected in a significant number of patients with mucosal melanoma [1]. The prognosis currently tends to be very poor, with a 5-year survival rates of $8.4 \%$, regardless of treatment, which

*We declare no conflicts of interest. usually involves primary surgery (conservative or radical) and adjuvant radiotherapy or systemic chemotherapy regimens [2,3]. The authors discuss three cases of gynecological melanoma and analyze clinical and prognostic aspects of this disease.

\section{CASES REPORT}

Cases 1 and 2: We describe two cases of 54-year-old and 60-year-old postmenopausal female patients with history of 2 months of abnormal genital bleeding. On vaginal examination there was a firm mass growth of size $3 \mathrm{~cm}$ attached to the right lateral wall of o the upper-third of the vagina. A biopsy of the vaginal tumor was taken, which revealed melanoma. Radiological assessment was performed. Abdominal and pelvic Ultrasound (US), CT (Figure 1) and magnetic resonance (MRI) confirmed the presence of invasive vaginal lesion of $5 \mathrm{~cm}$ located in the right lateral wall without lymph node involvement or distant metastasis.

Case 3: A female patient 43 years of age, with history of 4 months of abnormal genital bleeding. A gynecological examination showed a cervical polyp. The excision

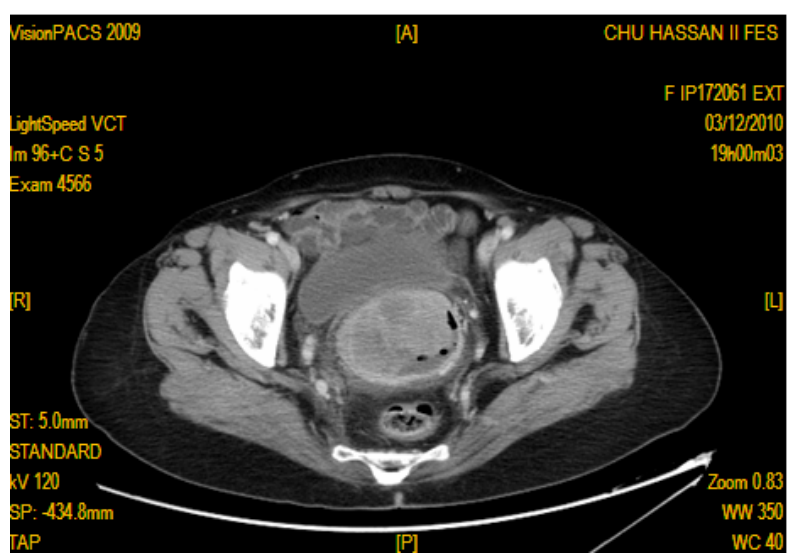

Figure 1. Invasive vaginal lesion located in the lateral wall. 
was performed which revealed melanoma. Magnetic resonance (MRI) (Figure 2) showed cervical tissular mass with infiltration of the anorectal wall without lymph nodes.

Microscopically, in the three cases, neoplasia was formed by nodules of broad cytoplasmic cells with poorly defined borders with pleomorphic nuclei with prominent nucleolar. These areas displayed transition with areas with fascicules of elongated cells of moderate cytoplasm. Immunohistochemical stains were positive for S-100 protein, Melan A (Figure 3), HMB 45 and CD117 (Figure 4) in neoplastic cells. CKAE1/AE3 and CEA were negative.

Surgery was not indicated given the advanced stage of disease.

The first patient received chemotherapy with a poor

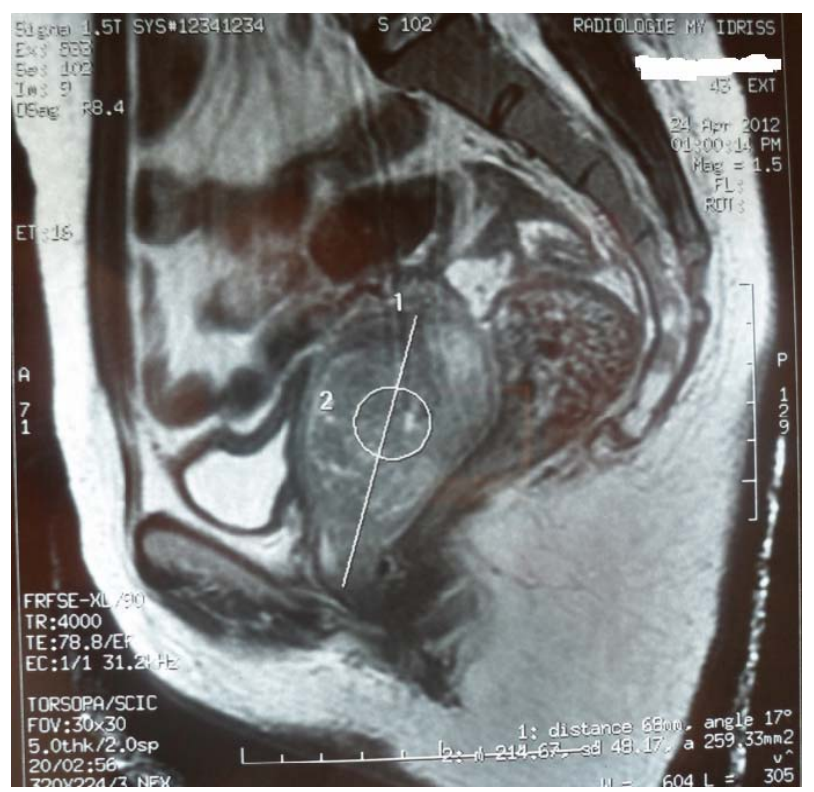

Figure 2. Cervical tissular mass with infiltration of the anorectal wall.

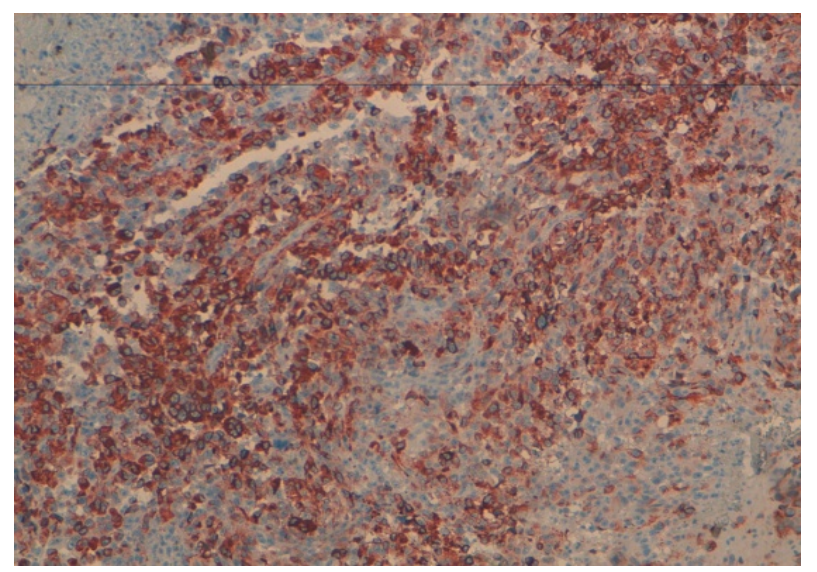

Figure 3. Immunohistochemical stains positivity for Melan A in neoplastic cells.

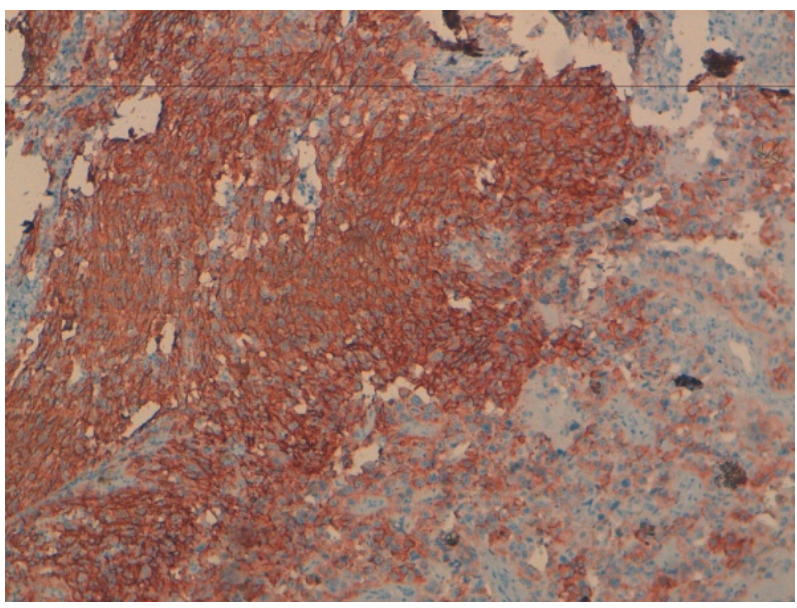

Figure 4. Immunohistochemical stains positivity for CD117 in neoplastic cells.

tolerance. Imatinib was administered as second line with evolution after three cure, then stabilization of the disease. She died after one year.

The other two patients received chemotherapy with dacarbazine. They had their third course with good development and good tolerance.

\section{DISCUSSION}

Malignant melanomas are generally found in areas of skin exposed to the sun, but can also be present in non exposed sites, such as genital tract and esophagus, among others [4]. The first case of malignant melanoma of the female genital tract (MMFG) was reported by Hewitt in 1861 [5].

Cervical melanoma arises from melanocytic cells of the cervix; in fact, the cervix epithelium is capable of forming the complete spectrum of melanocytic lesions, from benign lentigines to blue nevi to melanoma [6]. The usual form of presentation of primary melanoma of the cervix on physical examination is a polypoid exophytic mass, red, brown, grey, black, or blue in color [7], or a colorless in the case of amelanotic melanomas, which constitute up to $55 \%$ of cases at this anatomic site [8], presented with vaginal bleeding $[4,7,9,10]$. Age range varies from 20 to 78 years [7], being more common between 60 and 70 years [9]. Due to that the cervix is an unusual site for this type of neoplasm, the International Federation of Gynecology and Obstetrics (FIGO) staging system for cervical cancer is used [11], rather than the Clark and Breslow scales, because the FIGO staging system correlates better with the prognosis. Diagnosis of primary melanoma of the cervix entertains a high probability of being confused with another entity, due to the rarity of the disease [10]. Differential diagnosis between a primary cervical melanoma and a metastatic tumor is important because the latter can be part of a metastatic 
disease spreading to the cervix [9]. At the present moment there is no standard treatment for this disease, while there is no doubt that the surgical approach is the most usual and radical hysterectomy with or without pelvic lymphadenectomy and/or superior vaginectomy is reported most frequently $[4,9,12,13]$, some authors entertain doubts concerning survival if pelvic lymphadectomy is performed [12]. Although there is no enough information about the real role of negative margins in primary melanoma of the cervix, the primary surgery should have the purpose of obtaining negative margins [7]; some authors recommend 2-cm margins as minimum $[10,13]$. The role of radiotherapy (RT) has not been well established, but it has been demonstrated that RT reduces the tumor size $[4,9,11]$. The use of adjuvant pelvic RT is considered in the case of not obtaining a satisfactory surgical resection margins, when the parametrium is involved, or when lymph nodes are found to be involved [7]. Despite the low level of radiosensitivity exhibited by melanoma, the use of external or intracavitary RT is recommended after surgery, or palliatively when the tumor is inoperable because of its own conditions or those of the patient $[4,6,7,9]$.

Primary vaginal melanoma is also a very rare gynecologic malignancy and limited data are available on which to base recommendations for the primary management of patients. Our cases are middle-aged women with primary malignant melanoma of the vagina, $5 \mathrm{~cm}$ in diameter and localized in the right lateral wall with infiltration to the anterior rectal wall and anal sphincter without identified local spread and distant metastasis. There was clinical impression of an infiltration to the anterior rectal wall and anal sphincter without any clinically identified local spread and distant metastasis. The aim should have been to completely resect the tumor with tumor-free surgical margins and evaluate the related lymph nodes for tumor involvement in primary treatment. Miner et al. reported improved outcomes with surgical removal of macroscopic disease whenever possible [14]. There are several treatment options but none of them is proved to be standard approach. The spectrum of surgical therapy ranges from conservative surgery such as wide excision of the lesion or total vaginectomy to radical extirpation with en bloc removal of involved pelvic organs [14].

In gynecological melanoma, no chemotherapy regimes have been reported that substantially may reduce the possibility of recurrence. Dacarbazine is utilized in advanced disease, as the case for our patients, and it has been observed that up to $20 \%$ of patients may have response $[7,13]$. It has been proposed that the combination of cisplatin, bleomycin, and vinblastine can provoke a better response than the use of solely dacarbazine [9], while in other cases, the greater effectiveness of combining dacarbazine with vincristine and carmustine has been reported as well as immunotherapy utilizing local BCG or the transfusion of activated lymphocytes [6].

Average survival reported in the world literature of these patients ranges from 6 months to 14 years. The majority of them report that they succumb to the disease in the first 3 years after diagnosis [4]. Five-year survival after radical hysterectomy as only treatment is very low: less than $40 \%$ in stage I and $14 \%$ in stage II $[9,15]$.

There are no standard guidelines for adequate staging workup in mucosal melanoma. A thorough history and physical examination are extremely important and many experts recommend serum lactate dehydrogenase as well as axial imaging of brain, chest, abdomen, and pelvis and/or positron emission tomography (PET) scans to evaluate the stage and extent of disease at diagnosis. The staging system for mucosal melanoma has also not been well established. Because of the advanced presentation of most cases, Breslow depth alone seems to be of little use in staging of the majority of primary mucosal melanomas. Hence, oncologists use different systems to stage mucosal melanoma. Although some clinicians prefer a simple and practical staging system in which stage $\mathrm{I}$ is localized, stage II is regional (lymph node involvement), and stage III is distant disease, most use the American Joint Committee on Cancer (AJCC) staging system, referring to distant metastatic disease as stage IV. Establishment of an effective staging systembased on prognostic factors unique to mucosal melanoma would be beneficial [1].

In 2006 shed light on c-KIT genetic alterations in melanoma. That study showed that c-KIT amplification/ mutation occurred in $39 \%$ of mucosal melanomas, but none of non-chronic sun damage cutaneous melanoma cases. Mucosal melanoma has been shown to exhibit amplifications or increased copy numbers of the $4 q 12$ locus by comparative genomic hybridization. Mutations, including the activating K462E mutations of the c-KIT kinase domain, which are known to render sensitivity to imatinib (a tyrosine kinase inhibitor) in gastrointestinal stromal tumors, are also commonly seen in mucosal melanoma. The prevalence of these mutations in mucosal melanoma is $5 \%$ - $22 \%$ based on some studies [16-18], which is much lower than what was originally expected. Preclinical studies have shown sensitivity of c-KIT mutant mucosal melanoma, providing a rationale for studying imatinib in this melanoma type in clinical trials. Marked tumor regression was reported in a patient with metastatic mucosal melanoma who was treated with single-agent imatinib [19]. This observation has been substantiated by several other anecdotal reports, demonstrating objective responses in patients with advanced mucosal and acral melanoma after imatinib monotherapy [20,21]. A phase II trial investigating response to imatinib in patients with unresectable melanoma harboring 
somatic alterations of c-KIT is currently ongoing at six institutions, including Memorial Sloan-Kettering Cancer Center.

Primary malignant melanoma of female genital tract is a rare disease with a poor prognosis, especially if it is not detected in a timely fashion or if it is not treated correctly. To date, no consensus has been established concerning treatment of gynecologic primary Melanoma. But it is recommended that this be surgical, procuring the establishment of 2-cm margins, accompanied by radio- or chemo-therapy.

\section{REFERENCES}

[1] Seetharamu, N., Ott, P.A. and Pavlick, A.C. (2010) Mucosal melanomas: A case-based review of the literature. The Oncologist, 15, 772-781. doi:10.1634/theoncologist.2010-0067

[2] Irvin Jr, W.P., Bliss, S.A., Rice, L.W., Taylor Jr, P.T. and Andersen, W.A. (1998) Malignant melanoma of the vagina and locoregional control: Radical surgery revisited. Gynecologic Oncology, 71, 476-480. doi:10.1006/gyno.1998.5188

[3] Bonner, J.A., Perez-Tamayo, C., Reid, G.C., Roberts, J.A. and Morley, G.W. (1988) The management of vaginal melanoma. Cancer, 62, 2066-2072. doi:10.1002/1097-0142(19881101)62:9<2066::AID-CNC R2820620933>3.0.CO;2-D

[4] Siozos, C., Bhat, A., Lonsdale, R., Nieto, J.J. and Crocker, S.G. (2005) Malignant melanoma of the uterine cervix. Journal of Obstetrics and Gynaecology, 25, 826-827. doi:10.1080/01443610500338305

[5] Hewitt P. Sequel to a case of recurrent melanosis of both groins and back: The disease reappearing in the brain, heart, pancreas, liver and other organs. Lancet, 1861, 263-264.

[6] Gupta, R., Singh, S. and Mandal, A.K. (2005) Primary malignant melanoma of cervix-A case report. Indian Journal of Cancer, 42, 201-204.

[7] Piura, B. (2008) Management of primary melanoma of the female urogenital tract. The Lancet Oncology, 9, 973981. doi:10.1016/S1470-2045(08)70254-7

[8] Deshpande, A.H. and Munshi, M.M. (2001) Primary malignant melanoma of the uterine cervix: Report of a case diagnosed by cervical scrape cytology and review of the literature. Diagnostic Cytopathology, 25, 108-111. doi:10.1002/dc.2014

[9] Baruah, J., Roy, K.K., Kumar, S. and Kumar, L. (2009) A rare case of primary malignant melanoma of cervix. Archives of Gynecology and Obstetrics, 280, 453-456. doi:10.1007/s00404-008-0912-0

[10] An, J., Li, B., Wu, L., Lu, H. and Li, N. (2009) Primary malignant amelanotic melanoma of the female genital tract: Report of two cases and review of literature. Melanoma Research, 19, 267-270. doi:10.1097/CMR.0b013e32831993de

[11] Mousavi, A.S., Fakor, F., Nazari, Z., Ghaemmaghami, F., Hashemi, F.A. and Jamali, M. (2006) Primary malignant melanoma of the uterine cervix: Case report and review of the literature. Journal of Lower Genital Tract Disease, 10, 258-263. doi:10.1097/01.lgt.0000229564.11741.4e

[12] Wasef, W.R., Roberts, J.K. and Dixon, G.R. (1999) Primary malignant melanoma of the cervix uteri. Journal of Obstetrics and Gynaecology, 19, 673-674. doi:10.1080/01443619964094

[13] Cantuaria, G., Angioli, R., Nahmias, J., Estape, R. and Penalver, M. (1999) Primarymalignant melanoma of the uterine cervix: Case report and review of the literature. Gynecologic Oncology, 75, 170-174. doi:10.1006/gyno.1999.5491

[14] Miner, T.J., Delgado, R., Zeisler, J., et al. (2004) Primary vaginal melanoma: A critical analysis of therapy. Annals of Surgical Oncology, 11, 34-39. doi:10.1007/BF02524343

[15] Clark, K.C., Butz, W.R. and Hapke, M.R. (1999) Primary malignant melanoma of the uterine cervix: Case report with world literature review. International Journal of Gynecological Pathology 18, 265-273. doi:10.1097/00004347-199907000-00013

[16] Curtin, J.A., Busam, K., Pinkel, D., et al. (2006) Somatic activation of KIT in distinct subtypes of melanoma. Journal of Clinical Oncology, 24, 4340-4346. doi:10.1200/JCO.2006.06.2984

[17] Antonescu, C.R., Busam, K.J., Francone, T.D., et al. (2007) L576P KIT mutation in anal melanomas correlates with KIT protein expression and is sensitive to specific kinase inhibition. International Journal of Cancer, 121, 257-264. doi:10.1002/ijc.22681

[18] Torres-Cabala, C.A., Wang, W.L., Trent, J., et al. (2009) Correlation between KIT expression and KIT mutation in melanoma: A study of 173 cases with emphasis on the acral-lentiginous/mucosal type. Modern Pathology, 22, 1446-1456. doi:10.1038/modpathol.2009.116

[19] Hodi, F.S., Friedlander, P., Corless, C.L., et al. (2008) Major response to imatinib mesylate in KIT-mutated melanoma. Journal of Clinical Oncology, 26, 2046-2051. doi:10.1200/JCO.2007.14.0707

[20] Lutzky, J., Bauer, J. and Bastian, B.C. (2008) Dose-dependent, complete response to imatinib of a metastatic mucosal melanoma with a K642E KIT mutation. Pigment Cell \& Melanoma Research, 21, 492-493. doi:10.1111/j.1755-148X.2008.00475.X

[21] Jiang, X., Zhou, J., Yuen, N.K., et al. (2008) Imatinib targeting of KIT-mutant oncoprotein in melanoma. Clinical Cancer Research, 14, 7726-7732. doi:10.1158/1078-0432.CCR-08-1144 